\title{
A genome-wide study of DNA methylation modified by epigallocatechin-3-gallate in the CAL-27 cell line
}

\author{
LI-LI CHEN ${ }^{1,2}$, WEN-FEI HAN ${ }^{1}$, YING GENG $^{1}$ and JIAN-SHENG SU ${ }^{1}$ \\ ${ }^{1}$ Laboratory of Oral Biomedical Science and Translational Medicine, School of Stomatology, Tongji University, Shanghai 200072; \\ ${ }^{2}$ Department of Stomatology, Zhongshan Hospital, Xiamen University, Xiamen, Fujian 361004, P.R. China
}

Received June 6, 2014; Accepted March 3, 2015

DOI: $10.3892 / \mathrm{mmr} .2015 .4118$

\begin{abstract}
In order to gain greater understanding of the mechanisms underlying the effect of epigallocatechin-3-gallate (EGCG) on DNA methylation and its chemopreventative action in oral squamous cell carcinoma (OSCC), a genome-wide methylation and mRNA expression screen was performed in the CAL-27 cell line with and without EGCG $(100 \mu \mathrm{M})$ treatment. A total of 761 differentially methylated gene loci were identified following treatment with EGCG. Comparison of gene expression profiling in OSCC samples revealed 184 transcripts with a significant difference $(\mathrm{P}<0.05)$ and a fold change difference $>2$ compared with controls. Gene ontology analysis of differentially methylated loci and functional annotation of the differentially expressed genes indicated that the main pathways involved were metabolic, mitogen-activated protein kinase (MAPK), wnt, and cell cycle pathways. In conclusion, the present study indicates that EGCG can affect the methylation status and gene expression in the CAL-27 cell line. Additionally, the changes in several important signaling pathways may reveal the antitumor mechanism of EGCG.
\end{abstract}

\section{Introduction}

Green tea is one of the most popular beverages consumed worldwide, and its tumor-suppressive effects have been reported in various types of cancer, including human head and neck squamous cell carcinoma (1). Epigallocatechin-3-gallate (EGCG), the most abundant polyphenol in green tea, is considered to have the predominant anticancer and cancer-preventive effects (2).

EGCG inhibits carcinogen activity, tumorigenesis, proliferation and angiogenesis, and induces cell death. These effects are associated with modulation of reactive oxygen species (ROS) production and nuclear factor- $\kappa \mathrm{B}$, mitogen-activated

Correspondence to: Dr Jian-Sheng Su, Laboratory of Oral Biomedical Science and Translational Medicine, School of Stomatology, Tongji University, 399 Yanchang Middle Road, Shanghai 200072, P.R. China E-mail: jierduncll@126.com

Key words: epigallocatechin-3-gallate, epigenetics, cancer, methylation protein kinase (MAPK), epidermal growth factor receptor and insulin-like growth factor-I signaling pathways, affecting diverse processes, such as proliferation, differentiation, apoptosis, angiogenesis, metastasis and migration (3). In addition, EGCG could also induce epigenetic modification by inhibition of DNA methyltransferase activity and regulation of histone acetylation, leading to an upregulation of apoptosis (4).

Alterations in DNA methylation, including hypomethylation of oncogenes and hypermethylation of tumor suppressor genes, particularly the hypermethylation of promoter $\mathrm{CpG}$ islands are critical in cancer progression (5). Oral squamous cell carcinoma (OSCC) represents the eighth most common type of malignancy in males and the 13th most common type in females worldwide, accounting for $\sim 38 \%$ of all head and neck tumors (6). Previous studies have demonstrated that EGCG has cancer-preventative activity in OSCC development (7-9). Mechanistically, this may occur via epigenetic modulation. To date, several studies have investigated DNA methylation and EGCG $(10,11)$. These have predominantly focused on the ability of EGCG to inhibit DNA methyltransferase (DNMT), which leads to the reactivation of genes silenced by promoter or enhancer methylation, including $16^{\mathrm{INK} 4 \mathrm{a}}$, RAR $\beta$, MGMT, hMlH1, WIF-1 and hTERT $(12,13)$. Although these studies were not specific to the development of OSCC, they have indicated that EGCG is capable of affecting genes involved in carcinogenesis, via an epigenetic pathway.

To better understand the mechanisms underlying the effect of EGCG on DNA methylation and its chemopreventative action in OSCC, DNA methylation and mRNA expression profiling in CAL-27 cells treated with EGCG was analyzed.

\section{Materials and methods}

Cell culture and drug treatment. The CAL-27 human OSCC cell line, , was purchased from the American Type Culture Collection (Manassas, VA, USA). Cells were cultured in Dulbecco's modified Eagle's medium (Hyclone, GE Healthcare, Little Chalfont, UK) supplemented with $10 \%$ heat-inactivated fetal bovine serum (Hyclone, GE Healthcare), penicillin $\left(100 \mathrm{IU} \mathrm{ml}^{-1}\right)$ and streptomycin $\left(100 \mu \mathrm{g} \mathrm{ml}^{-1}\right)$, and maintained at $37^{\circ} \mathrm{C}$ in a $5 \% \mathrm{CO}_{2}$ atmosphere. EGCG (14) was obtained from Sigma Aldrich (Carlsbad, CA, USA) (E4143 EGCG, $\geq 95 \%$ ) and freshly prepared each time prior to use. For 
microarray analysis, cells were left untreated or were treated with $100 \mu \mathrm{M}$ EGCG for $24 \mathrm{~h}$, then genomic DNA and RNA were isolated.

Cell proliferation assay. The effect of EGCG on CAL-27 cell growth was assessed by an MTT cell proliferation and cytotoxicity detection kit (KeyGen Biotech, Nanjing, China). Cells $\left(7 \times 10^{4}\right)$ were seeded onto 96-well plates and grown to $80 \%$ confluence prior to treatment with the indicated concentrations of EGCG $(0-200 \mu \mathrm{M})$. After $24 \mathrm{~h}$, cell viability was determined by measuring absorbance at $490 \mathrm{~nm}$ using a microplate reader (Bio-Rad, Richmond, CA, USA). All assays were performed in triplicate.

Genome-wide methylation assay and statistical analysis. The Infinium II Methylation assay (Illumina, San Diego, CA, USA) was used to detect $27,578 \mathrm{CpG}$ sites genome-wide, spanning 14,495 genes. Illumina chip technology quantifies methylation levels at specific loci within the genome. Following bisulfite treatment, the unmethylated cytosine is converted into uracil and methylated cytosine remains unchanged. Following hybridization with the methylation-specific probe or the non-methylation probe, single-base extension with labelled dideoxynucleotides is performed. Finally, the fluorescence intensities of the methylated and unmethylated signals are measured at each targeted cytosine position to estimate DNA methylation level. Genomic DNA was extracted using the Genomic DNA Mini Preparation kit with Spin Columns (Beyotime Biotech., Jiangsu, China). Bisulfite conversion of genomic DNA from EGCG-treated $(n=3)$ and control $(n=3)$ samples, was performed using the Zymo EZ DNA methylation kit (Illumina). Samples were run using the automated process on the Infinium methylation BeadChips, in accordance with the manufacturer's instructions. Data were analyzed using BeadStudio Gene Expression Module v3.4 (Illumina).

All differential methylation analysis algorithms compared a group of samples with EGCG treatment to a control group. This comparison was made using the following error models: Illumina Custom Model. P-values were calculated using the following formula:

$$
p=z\left(\frac{\left|\beta_{\text {cond }}-\beta_{r e f}\right|}{\frac{s_{r e f}^{2}}{N_{r e f}}+\frac{s_{\text {cond }}^{2}}{N_{\text {cond }}}}\right) \quad \beta=\left(\frac{\operatorname{Max}(C y 5,0)}{\operatorname{Max}(C y 3,0)+\operatorname{Max}(C y 5,0)+100}\right)
$$

Where $\mathrm{z}$ is the two-sided tail probability of the standard normal distribution; $\mathrm{S}_{\text {ref }}$ and $\mathrm{S}_{\text {cond }}$ are standard deviations of probe signals; and $\mathrm{N}_{\text {ref }}$ and $\mathrm{N}_{\text {cond }}$ denote the number of samples in the reference (treatment without EGCG) and condition (treatment with EGCG) groups, respectively. $\beta=$ methylated signal/(unmethylated signal+methylated signal+100). To identify possible cellular functions of these genes, the Gene Ontology analysis tool AmiGO was used (amigo.geneontology. org/cgi-bin/amigo/browse.cgi) (15).

Gene expression and statistical analysis. Total RNA was extracted using TRIzol reagent (Invitrogen Life Technologies, Carlsbad, CA, USA). The concentration of RNA was determined by NanoDrop 8000 (Thermo Fisher Scientific, Inc., Wilmington, DE, USA). For amplification and labeling of the RNA with

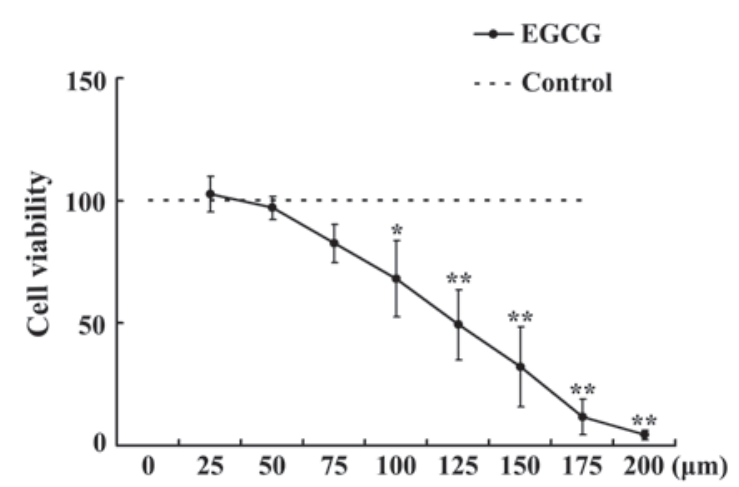

Figure 1. Effect of various doses of EGCG on CAL-27 cell proliferation after 24 h exposure. ${ }^{*} \mathrm{P}<0.05,{ }^{* *} \mathrm{P}<0.01$ vs. control.

the Illumina TotalPrep RNA Amplification kit, 200 ng RNA from each sample was used. The Illumina Sentrix arrays were processed according to the manufacturer's instructions. Slides were scanned immediately using Illumina BeadStation iScan. Data were analyzed using GenomeStudio Gene Expression Module v1.0 (Illumina).

All differential expression algorithms compared a group of samples with EGCG treatment with a control group. The comparison was performed using unpaired Welch's t-test for unequal variance. Genes of which expression was significantly different between the compared groups were selected based on a fold change $>2$ in gene expression as determined by a $\mathrm{t}$-test, and a $\mathrm{P}<0.05$ and corrected for multiple testing using the Benjamin-Hochberg method.

\section{Results}

Effect of EGCG on CAL-27 cell proliferation. The effect of various doses of EGCG on CAL-27 cell proliferation was investigated after $24 \mathrm{~h}$ exposure. As shown in Fig. 1, EGCG treatment significantly inhibited cell growth in a concentration-dependent manner. Inhibition of proliferation was clearly observed at a concentration of $100 \mu \mathrm{M}$ EGCG, and this concentration was therefore utilized in methylation profiling experiments

Genome-wide differential DNA methylation profiling in EGCG-treated cells. To identify loci that were differentially methylated in CAL-27 cells in response to treatment with EGCG, the Infinium II Methylation assay was used, which interrogated 27,578 loci, covering $>14,000$ genes. Comparison of methylation profiles between EGCG-treated and control samples identified 677 genes $(\mathrm{P}<0.05)$ and 84 genes $(\mathrm{P}<0.01)$ that were differentially methylated. Of the 84 genes altered in response to EGCG treatment, 57 were hypermethylated and 24 were hypomethylated (Fig. 2A and B). To identify possible cellular functions of these genes, gene ontology (GO) analysis was performed. Biological functions of hypermethylated genes include transport, cell cycle, transduction, oxidative processes and apoptosis, whereas hypomethylated gene loci were enriched for genes involved in apoptosis, transduction, oxidative processes and cell adhesion (Fig. 2A and B). 

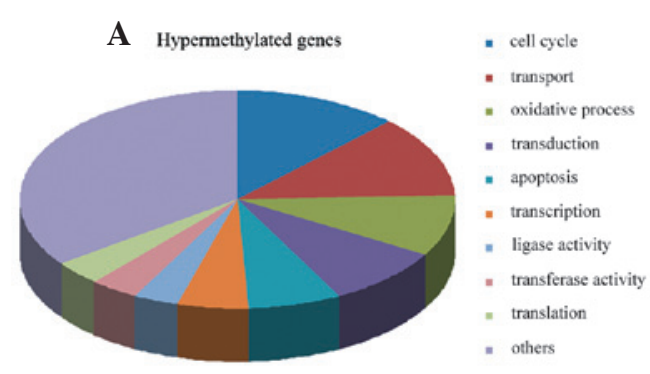

C

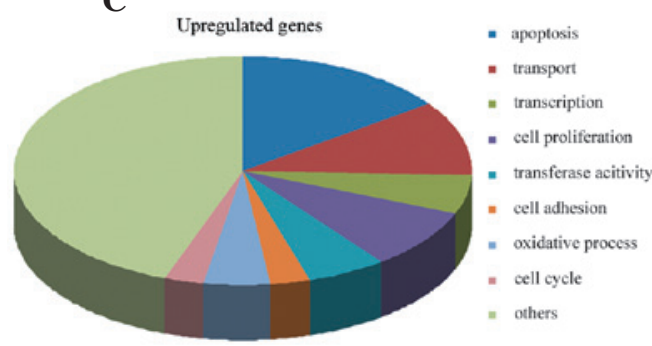

B

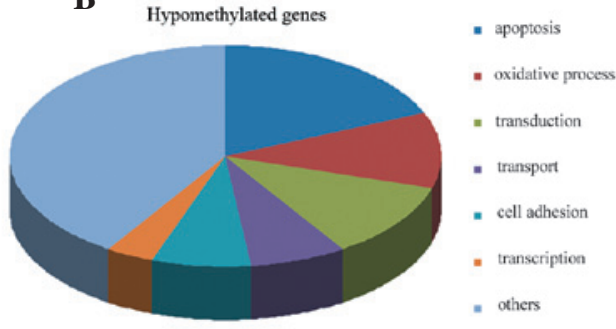

D

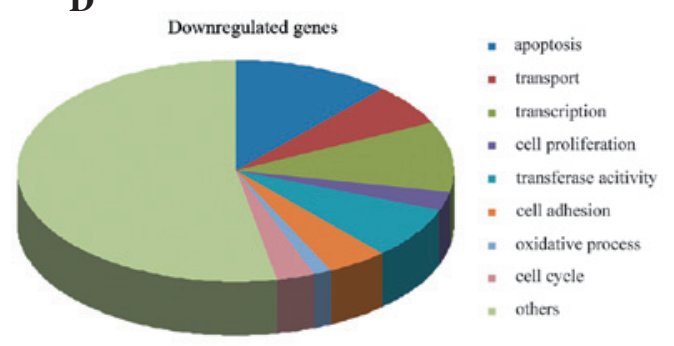

Figure 2. GO analysis (Biological Process) of different genes between EGCG-treated and control CAL-27 cell lines. (A) hypermethylated genes, (B) hypomethylated genes; (C) upregulated genes; (D) downregulated genes.

A

A

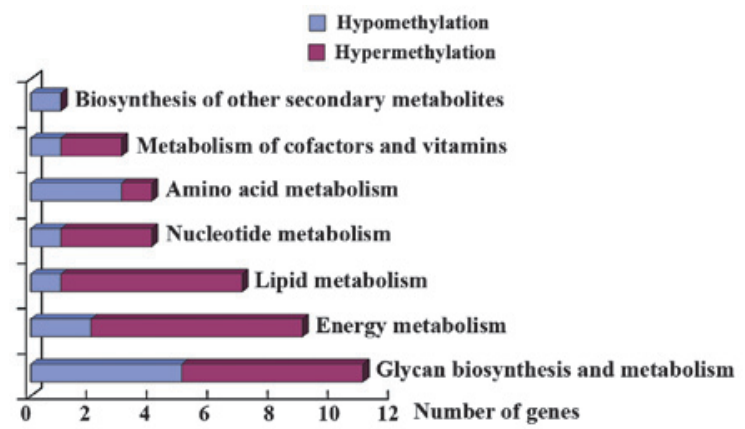

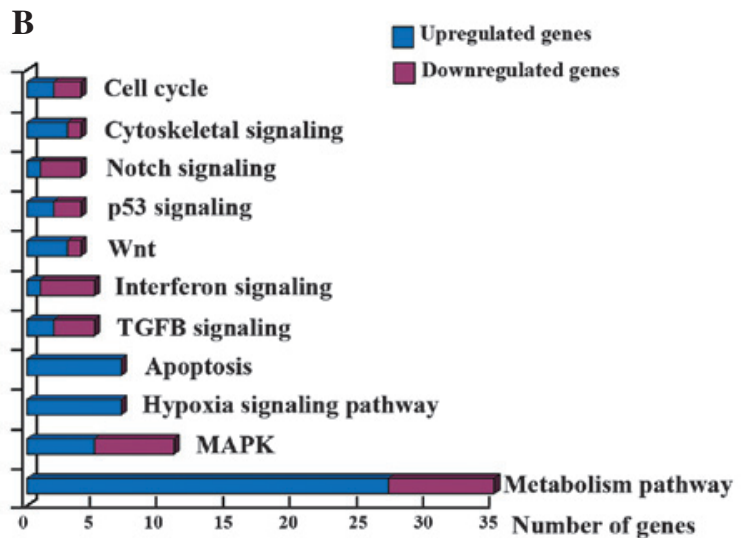

Figure 3. Pathway analysis of different genes between EGCG-treated and control CAL-27 cell lines. (A) Hypermethylated/hypomethylated genes and (B) upregulated/downregulated genes.
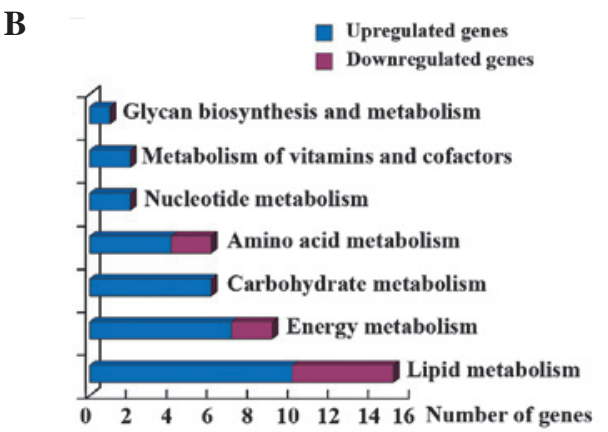

Figure 4. Metabolic pathway analysis of different genes between EGCG-treated and control CAL-27 cell line. (A) Hypermethylated/hypomethylated genes and (B) upregulated/downregulated genes.

Differentially methylated genes are enhanced for specific signaling pathways. To identify signaling pathways associated with EGCG treatment, pathway analyses were performed. Of the 677 genes $(\mathrm{P}<0.05)$ differentially methylated following EGCG treatment, 229 genes were associated with signaling pathways. The top 10 enriched pathways were: Metabolic pathways, cell cycle/mitotic, axon guidance, MAPK signaling, cytokine-cytokine receptor interaction, signaling by nerve growth factor, protein processing in endoplasmic reticulum, signaling by G-protein-coupled receptor, Wnt signaling 
pathway and cell cycle (Fig. 3A). Analysis of metabolic pathway genes affected by EGCG treatment identified an enrichment of genes involved in glycan biosynthesis and metabolism, energy metabolism and lipid metabolism (Fig. 4A). The other 84 genes $(\mathrm{P}<0.01)$ were also analyzed but no enriched pathways were identified.

Differential gene RNA expression. Whole-genome gene expression analysis was performed on RNA samples isolated from CAL-27 cells with and without EGCG treatment. One hundred and eighty-four transcripts showed a fold change difference $>2$ in gene expression after EGCG treatment in CAL-27 cells from 47,317 detected probes in the array. One hundred and fifteen transcripts were upregulated and 69 downregulated among the 184 transcripts that were significantly changed after EGCG treatment. Up- and downregulated genes were involved in the following biological functions: Apoptosis, transport, transcription, cell proliferation, transferase activity, cell adhesion, oxidative processes and the cell cycle (Fig. 2C and D).

Pathway analyses indicated that the main pathways associated with the differentially expressed genes were: Metabolism pathway, MAPK, hypoxia signaling pathway, apoptosis, transforming growth factor $\beta$ signaling, interferon signaling, Wnt, p53 signaling, Notch signaling, cytoskeletal signaling and cell cycle (Fig. 3B).

Analysis of metabolic pathway genes affected by EGCG treatment identified an enrichment of genes involved in lipid, energy and carbohydrate metabolism (Fig. 4B).

\section{Discussion}

A number of studies using cell culture and animal models have suggested that EGCG is a prospective candidate for use in the chemoprevention of cancer (16-22). Anticancer effects of EGCG include: Inhibition of carcinogen activity and tumorigenesis; inhibition of tumor proliferation and angiogenesis; inhibition of tumor migration and invasion; and induction of cell death (23). These effects have also been found in OSCC, including in the CAL-27 cell line (24-26). However, to the best of our knowledge, the mechanisms underlying EGCG action have not been clarified. DNA methylation is the most extensively investigated epigenetic modification. Hypermethylation on the DNA molecule limits the binding of transcription factors to promoters, resulting in the recruitment of additional proteins and gene silencing (27). This methylation is mediated by DNMT. EGCG is known to be an inhibitor of DNMT by direct inhibitory interaction with the catalytic site of DNMT (28). Several studies have found that EGCG reverses the methylation-mediated downregulation of the tumor suppressors and then reduces cell growth and colony formation (29). However, few studies have investigated the effect of EGCG on global methylation. The demethylation effect of EGCG on the matrix metalloproteinase inhibitor, RECK, is the only report of EGCG modulating DNA methylation in oral carcinoma cells (30).

In the present study, EGCG treatment significantly inhibited the proliferation of CAL-27 cells after $24 \mathrm{~h}$, in a concentration-dependent manner. These results are consistent with our previous findings that, at $100 \mu \mathrm{M}$ EGCG, the survival rate of CAL-27 cells rapidly declined; however, the survival rate of human gingival fibroblasts was not affected, even after $100 \mu \mathrm{M}$ EGCG treatment for $72 \mathrm{~h}$. Hence, $100 \mu \mathrm{M}$ EGCG was used to treat CAL-27 cells, which does not harm normal oral cells but inhibits the growth of oral cancer cells.

This study represents the first genome-wide methylation analysis of genes affected by EGCG. EGCG treatment of CAL-27 cells leads to hypermethylation and hypomethylation of gene loci, while EGCG treatment was associated with higher levels of hypermethylation compared with hypomethylation (57 vs. 27 genes). Whole genome expression analysis indicated that the expression of 184 transcripts was altered. Although the majority of genes with altered methylation showed no significant changes in expression, the function and pathway analyses indicated the anti-tumor effects of EGCG. Analysis of genes with changed methylation status revealed their functions are involved in the regulation of the cell cycle, transport, oxidative processes, apoptosis, transcription, transferase activity and cell adhesion. These functions are consistent with the analysis of genes with changed expression levels and strongly indicate the anticancer properties of EGCG.

Integrated analysis of DNA methylation and mRNA expression showed that four pathways were significantly changed by EGCG treatment: Metabolism, MAPK, Wnt and cell cycle pathways. A large portion of genes that were altered upon treatment with EGCG were involved in metabolism pathways. The ability of EGCG to affect metabolism may explain why it is considered to be beneficial in the prevention and/or treatment of cardiovascular and metabolic diseases, such as obesity and diabetes mellitus (31). Given that metabolism pathways are essential for cancer cells, they may be an important target from EGCG, contributing to its inhibitory effect on tumorigenesis.

MAPKs are composed of extracellular signal-regulated kinase, p38 MAPK, and c-Jun N-terminal kinase, and the deregulation of MAPK cascades contributes to cancer. MAPK signaling has previously been identified as a target for cancer prevention by EGCG (32), the present data show that it may be regulated via an epigenetic mechanism.

Aberrant regulation of the Wnt signaling pathway exhibits an important role in cancer biology. Kim et al (33) found that EGCG inhibits Wnt signaling and the Wnt target gene c-MYC in breast cancer cells by inducing the HBP1 transcriptional repressor and inhibiting aspects of invasive breast cancer. According to the data of the present study, EGCG changed the methylation status of CSNK1E, CSNK2A1, LRP6, MYC, NFATC4, SMSD4, TCF7 and TCF7L1, and altered gene expression of ANGPTL4, DAB2, NDRG1 and CXXC5, which was associated with the Wnt signaling pathway.

The cell cycle is a series of events that takes place in a cell leading to its division and duplication. Regulation of the cell cycle involves processes crucial to the survival of a cell, including the detection and repair of genetic damage as well as the prevention of uncontrolled cell division. EGCG downregulated the cell cycle regulatory proteins CCND1 and PPP2R2B, and upregulated CDKN1C. This may have occurred via epigenetic mechanisms. Other pathways, such as apoptosis and p53 signaling, are classical antitumor pathways, their dysregulation following EGCG treatment strongly indicated the antitumorigenic activity of EGCG. 
In conclusion, to the best of our knowledge, the present study reported for the first time the genome-wide analysis of promoter methylation and expression profiling in the OSCC cell line treated with EGCG. Additionally, the changes in several important signaling pathways may reveal the antitumor mechanism of EGCG.

\section{Acknowledgements}

This study was sponsored by the National Science Foundation of China (grant no. NFSC-81371949, 30970726), and Shanghai (grant nos. 114119a3700 and 13411951201).

\section{References}

1. Yang CS, Wang X, Lu G and Picinich SC: Cancer prevention by tea: animal studies, molecular mechanisms and human relevance. Nat Rev Cancer 9: 429-439, 2009.

2. Shimizu M, Shirakami Y and Moriwaki H: Targeting receptor tyrosine kinases for chemoprevention by green tea catechin, EGCG. Int J Mol Sci 9: 1034-1049, 2008.

3. Khan N, Afaq F, Saleem M, Ahmad N and Mukhtar H: Targeting multiple signaling pathways by green tea polyphenol (-)-epigallocatechin-3-gallate. Cancer Res 66: 2500-2505, 2006.

4. NandakumarV,VaidMandKatiyarSK:(-)-Epigallocatechin-3-gallate reactivates silenced tumor suppressor genes, Cip1/p21 and p16INK4a, by reducing DNA methylation and increasing histones acetylation in human skin cancer cells. Carcinogenesis 32: 537-544, 2011.

5. Lund AH and van Lohuizen M: Epigenetics and cancer. Genes Dev 18: 2315-2335, 2004

6. Parkin DM, Bray F, Ferlay J and Pisani P: Global cancer statistics, 2002. CA Cancer J Clin 55: 74-108, 2005.

7. Hwang YS, Park KK and Chung WY: Epigallocatechin-3 gallate inhibits cancer invasion by repressing functional invadopodia formation in oral squamous cell carcinoma. Eur J Pharmacol 715 : 286-295, 2013.

8. Chen PN, Chu SC, Kuo WH, Chou MY, Lin JK and Hsieh YS: Epigallocatechin-3 gallate inhibits invasion, epithelial-mesenchymal transition and tumor growth in oral cancer cells. J Agric Food Chem 59: 3836-3844, 2011.

9. Ho YC, Yang SF, Peng CY, Chou MY and Chang YC: Epigallocatechin-3-gallate inhibits the invasion of human oral cancer cells and decreases the productions of matrix metalloproteinases and urokinase-plasminogen activator. J Oral Pathol Med 36: 588-593, 2007.

10. Zhang Y, Wang X,Han L, Zhou Y and Sun S: Green tea polyphenol EGCG reverse cisplatin resistance of A549/DDP cell line through candidate genes demethylation. Biomed Pharmacother 69: 285-290, 2015

11. Jin H, Chen JX, Wang H, Lu G, Liu A, Li G, Tu S, Lin Y and Yang CS: NNK-induced DNA methyltransferase 1 in lung tumorigenesis in $\mathrm{A} / \mathrm{J}$ mice and inhibitory effects of (-)-epigallocatechin-3-gallate. Nutr Cancer 67: 167-176, 2015

12. Lee WJ, Shim JY and Zhu BT: Mechanisms for the inhibition of DNA methyltransferases by tea catechins and bioflavonoids. Mol Pharmacol 68: 1018-1030, 2005.

13. Gao Z, Xu Z, Hung MS, Lin YC, Wang T, Gong M, Zhi X, Jablon DM and You L: Promoter demethylation of WIF-1 by epigallocatechin-3-gallate in lung cancer cells. Anticancer Res 29: 2025-2030, 2009

14. Lai SM, Gu JY, Huang BH, Chang CM and Lee WL: Preparative separation and purification of epigallocatechin gallate from green tea extracts using a silica adsorbent containing $\beta$-cyclodextrin. J Chr omatogr B Analyt Technol Biomed Life Sci 887-888: 112-121, 2012.

15. An IS, An S, Park S, Lee SN and BaeS: Involvement of microRNAs in epigallocatechin gallate-mediated UVB protection in human dermal fibroblasts. Oncol Rep 29: 253-259, 2013.

16. Berletch JB, Liu C, Love WK, Andrews LG, Katiyar SK and Tollefsbol TO: Epigenetic and genetic mechanisms contribute to telomerase inhibition by EGCG. J Cell Biochem 103: 509-519, 2008.
17. $\mathrm{Xu} Q$, Yang $\mathrm{CH}$, Liu Q, Jin XF, Xu XT, Tong JL, Xiao SD and Ran ZH: Chemopreventive effect of epigallocatechin-3-gallate (EGCG) and folic acid on the N-methyl-N'-nitro-N-nitrosoguanidine (MNNG)-induced gastrointestinal cancer in rat model. J Dig Dis 12: 181-187, 2011.

18. Li GX, Chen YK, Hou Z, Xiao H, Jin H, Lu G, Lee MJ, Liu B, Guan F, Yang Z, Yu A, et al: Pro-oxidative activities and dose-response relationship of (-)-epigallocatechin-3-gallate in the inhibition of lung cancer cell growth: a comparative study in vivo and in vitro. Carcinogenesis 31: 902-910, 2010.

19. Shen X, Zhang Y, Feng Y, Zhang L, Li J, Xie YA and Luo X: Epigallocatechin-3-gallate inhibits cell growth, induces apoptosis and causes $\mathrm{S}$ phase arrest in hepatocellular carcinoma by suppressing the AKT pathway. Int J Oncol 44: 791-796, 2014

20. Lim YC and Cha YY: Epigallocatechin-3-gallate induces growth inhibition and apoptosis of human anaplastic thyroid carcinoma cells through suppression of EGFR/ERK pathway and cyclin B1/CDK1 complex. J Surg Oncol 104: 776-780, 2011.

21. Maruyama T, Murata S, Nakayama K, Sano N, Ogawa K, Nowatari T, Tamura T, Nozaki R, Fukunaga K and Ohkohchi N: (-)-Epigallocatechin-3-gallate suppresses liver metastasis of human colorectal cancer. Oncol Rep 31: 625-633, 2014.

22. Hsu YC and Liou YM: The anti-cancer effects of (-)-epigalocathine-3-gallate on the signaling pathways associated with membrane receptors in MCF-7 cells. J Cell Physiol 226: 2721-2730, 2011.

23. Singh BN, Shankar S and Srivastava RK: Green tea catechin, epigallocatechin-3-gallate (EGCG): mechanisms, perspectives and clinical applications. Biochem Pharmacol 82: 1807-1821, 2011.

24. Liu X, Zhang DY, Zhang W, Zhao X, Yuan C and Ye F: The effect of green tea extract and EGCG on the signaling network in squamous cell carcinoma. Nutr Cancer 63: 466-475, 2011.

25. Lin HY, Hou SC, Chen SC, Kao MC, Yu CC, Funayama S, Ho CT and Way TD: (-)-Epigallocatechin gallate induces Fas/CD95-mediated apoptosis through inhibiting constitutive and IL-6-induced JAK/STAT3 signaling in head and neck squamous cell carcinoma cells. J Agric Food Chem 60: 2480-2489, 2012.

26. Chang CM, Chang PY, Tu MG, Lu CC, Kuo SC, Amagaya S, Lee CY, Jao HY, Chen MY and Yang JS: Epigallocatechin gallate sensitizes CAL-27 human oral squamous cell carcinoma cells to the anti-metastatic effects of gefitinib (Iressa) via synergistic suppression of epidermal growth factor receptor and matrix metalloproteinase-2. Oncol Rep 28: 1799-1807, 2012.

27. Ling Y, Zhang C, Xu Y, Zhu J, Zhu C, Lu M, Liu Y and Zhou T: Promoter methylation-associated silencing of p27kip1 gene with metastasis in esophageal squamous cell carcinoma. Mol Med Rep 9: 1075-1079, 2014.

28. Lee WJ, Shim JY and Zhu BT: Mechanisms for the inhibition of DNA methyltransferases by tea catechins and bioflavonoids. Mol Pharmacol 68: 1018-1030, 2005.

29. Fang MZ, Wang Y, Ai N, Hou Z, Sun Y, Lu H, Welsh W and Yang CS: Tea polyphenol (-)-epigallocatechin-3-gallate inhibits DNA methyltransferase and reactivates methylation-silenced genes in cancer cell lines. Cancer Res 63: 7563-7570, 2003.

30. Kato K, Long NK, Makita H, Toida M, Yamashita T, Hatakeyama D, Hara A, Mori H and Shibata T: Effects of green tea polyphenol on methylation status of RECK gene and cancer cell invasion in oral squamous cell carcinoma cells. Br J Cancer 99: 647-654, 2008.

31. Thielecke $\mathrm{F}$ and Boschmann M: The potential role of green tea catechins in the prevention of the metabolic syndrome-a review. Phytochemistry 70: 11-24, 2009.

32. Kim HS, Kim MH, Jeong M, Hwang YS, Lim SH, Shin BA, Ahn BW and Jung YD: EGCG blocks tumor promoter-induced MMP-9 bexpression via suppression of MAPK and AP-1 activation in human gastric AGS cells. Anticancer Res 24: 747-753, 2004.

33. Kim J,Zhang X, Rieger-Christ KM, Summerhayes IC, Wazer DE, Paulson KE and Yee AS: Suppression of Wnt signaling by the green tea compound (-)-epigallocatechin 3-gallate (EGCG) in invasive breast cancer cells. Requirement of the transcriptional repressor HBP1. J Biol Chem 281: 10865-10875, 2006. 